\title{
Téoros
}

Revue de recherche en tourisme

\section{La représentation de la femme sur les affiches touristiques balnéaires}

\author{
Le cas de la Normandie (1880-1960)
}

Christophe Pécout, Anaïs Bohuon et Ludovic Birot

Volume 29, numéro 2, 2010

Tourisme et femmes

URI : https://id.erudit.org/iderudit/1024877ar

DOI : https://doi.org/10.7202/1024877ar

Aller au sommaire du numéro

Éditeur(s)

Université du Québec à Montréal

ISSN

0712-8657 (imprimé)

1923-2705 (numérique)

Découvrir la revue

Citer cet article

Pécout, C., Bohuon, A. \& Birot, L. (2010). La représentation de la femme sur les affiches touristiques balnéaires : le cas de la Normandie (1880-1960). Téoros, 29(2), 112-118. https://doi.org/10.7202/1024877ar
Résumé de l'article

Le XIX ${ }^{\mathrm{e}}$ siècle marque l'avènement du tourisme balnéaire en Normandie. D'abord marqué par le caractère thérapeutique des bains de mer, le littoral normand devient un lieu de villégiature dédié aux loisirs mondains. Afin de promouvoir leur site et de donner le désir de rivage, les stations communiquent sur leurs atouts touristiques. Pour ce faire, l'affiche devient le média incontournable. Au sein de cette communication touristique, l'image de la femme prédomine. Principale actrice de ce tourisme, elle est mise en scène sous différents visages : en mondaine, en sportive ou en baigneuse. Or, cette représentation ne témoigne pas de sa réalité sociale. Aussi, cette femme idéalisée et fantasmée répond principalement à des enjeux économiques et publicitaires : il s'agit de vendre du rêve à travers les charmes féminins. 


\title{
La représentation de la femme sur les affiches touristiques balnéaires Le cas de la Normandie (1880-1960)
}

\author{
Christophe PÉCOUT \\ Équipe de recherche septentrionale en sciences du sport \\ Université de Lille 2, faculté des sciences du sport \\ et de l'éducation physique \\ christophe.pecout@cegetel.net
}

\author{
Anaïs BOHUON \\ Laboratoire sports, politiques et transformations sociales \\ Université Paris XI \\ anaisb1981@yahoo.fr
}

\author{
Ludovic BIROT \\ Équipe de recherche septentrionale en sciences du sport \\ Université de Lille 2, faculté des sciences du sport \\ et de l'éducation physique \\ ludovic.birot@univ-lille2.fr
}

\begin{abstract}
RÉSUMÉ: Le XIXe siècle marque l'avènement du tourisme balnéaire en Normandie. D'abord marqué par le caractère thérapeutique des bains de mer, le littoral normand devient un lieu de villégiature dédié aux loisirs mondains. Afin de promouvoir leur site et de donner le désir de rivage, les stations communiquent sur leurs atouts touristiques. Pour ce faire, l'affiche devient le média incontournable. Au sein de cette communication touristique, l'image de la femme prédomine. Principale actrice de ce tourisme, elle est mise en scène sous différents visages : en mondaine, en sportive ou en baigneuse. Or, cette représentation ne témoigne pas de sa réalité sociale. Aussi, cette femme idéalisée et fantasmée répond principalement à des enjeux économiques et publicitaires : il s'agit de vendre du rêve à travers les charmes féminins.
\end{abstract}

Mots-clés: Tourisme, Normandie, station balnéaire, femme, affiche.

Jusqu'au début du XIX ${ }^{e}$ siècle, le littoral demeure un lieu de travail, consacré à la pêche, mais en aucun cas un lieu de plaisir. Aussi, les habitations du littoral tournent généralement le dos à la mer. Deux modes vont faire naître l'intérêt pour le littoral : la médecine et l'aventure. Les médecins, d'abord en Angleterre, puis en Europe, décident de soulager leurs contemporains de la dégradation sanitaire des villes liée à la Révolution industrielle et notamment à la pollution de l'air. Dans les années 1730, un établissement de bains est construit à Brighton qui, en accueillant les familles royales, devient la première des stations balnéaires. Ce phénomène de médicalisation par les bains se développe tout le long du littoral de la mer du Nord : Doberan en Allemagne, Ostende en Belgique (Pic, 2009).

La France est touchée par cette mode. Ainsi dès le début du XIX ${ }^{\mathrm{e}}$ siècle, la côte normande sur laquelle viennent séjourner de nombreux aristocrates anglais s'impose comme une destination touristique. Le littoral normand devient alors un territoire à explorer où s'entremêlent les paysages grandioses (les falaises d'Etretat ou de Dieppe) et pittoresques mis en valeur par les peintres impressionnistes. Cependant, le littoral devient surtout un espace thérapeutique à travers la construction d'établissements de bains : Dieppe en 1822, Trouville en
1837, Honfleur en 1838, etc. C'est la naissance des bains de mer et du tourisme balnéaire. Devant cet afflux estival, les villages de pêcheurs se transforment progressivement en stations balnéaires, c'est-à-dire en communes vivant uniquement du tourisme lié à la mer.

Une fois fondées et lancées, ces stations ont un double objectif : promouvoir leur site pour attirer les touristes et donc générer de l'argent. La promotion et la communication sont par conséquent dès l'origine au cœur du processus touristique balnéaire. C'est pourquoi des guides touristiques sont imprimés à partir des années 1850. Écrits par des érudits régionaux, ces guides racontent l'histoire de la région, indiquent les itinéraires et les routes, décrivent les paysages pittoresques et expliquent les mœurs locales. Par contre, à partir des années 1880, l'affiche va se substituer au guide. La puissance de l'image et la multiplicité de ce support offrent, il est vrai, de nouvelles possibilités aux promoteurs des stations. D'ailleurs, la fonction de l'affiche est simple : séduire les touristes en présentant de manière sublimée les atouts de la station (paysages, plage, hôtels, casino, sport, etc.) Ce remodelage de la réalité vise à donner l'envie du voyage et le désir de rivage (Corbin, 1990). $\mathrm{Au}$ sein de cette propagande iconographique, un personnage prédomine : la femme. 
Tour à tour mise en scène sous différents visages, la femme s'incarne en mondaine, en sportive ou en baigneuse. Comment alors expliquer cette omniprésence féminine sur ces affiches balnéaires? Et surtout pourquoi l'absence quasi systématique de l'homme alors que celui-ci domine cette société du $\mathrm{XX}^{\mathrm{e}}$ siècle? Quelle femme veut-on montrer aux touristes? Le fait de la représenter le plus souvent dénudée traduit-il la réalité sociale des femmes ou bien n'est-elle qu'un argument commercial? Ce monde balnéaire dépeint, affranchi des codes, au sein duquel les femmes s'exhibent n'est-il qu'une image fantasmée et idéalisée? Autrement dit, ce tourisme balnéaire par ses pratiques sociales et culturelles (baignade, sports, mode, mondanités) participe-t-il à l'évolution des mœurs concernant la femme ou bien n'est-il qu'une manière illusoire de faire croire aux femmes qu'elles ont la possibilité de se libérer des carcans sociaux et moraux de l'époque?

Pour mieux comprendre et expliquer le phénomène, nous avons étudié près de 100 affiches touristiques liées à la Normandie éditées entre 1880 et 1960. Ces dates charnières correspondent à l'âge d'or de l'affiche touristique puisque que c'est à partir de 1880 qu'elle se massifie et disparaît progressivement à l'aube des années 1960 pour laisser place au nouveau média qu'est la télévision. Nos affiches ont été récoltées au musée Eugène Boudin de Honfleur, au musée Montebello de Trouville, au château-musée de Dieppe ainsi qu'aux archives départementales du Calvados. À partir de ce corpus, nous avons mené une analyse historique permettant de contextualiser l'affiche, une analyse sémiologique révélant les signes touristiques caractéristiques du monde balnéaire et une analyse graphique de l'affiche.

Les résultats de nos analyses s'organiseront en trois temps. Dans un premier temps, nous commencerons par interroger le support pour apprécier en quoi l'affiche devient le principal support de promotion du tourisme à cette époque. Dans un second temps, nous montrerons comment la mondaine devient la figure emblématique du tourisme balnéaire. Dans un dernier temps, nous traiterons de l'évolution de la représentation de la baigneuse - toujours en filigrane en réalité même si représentée différemment, la mondaine — passant de la simple baigneuse à la femme érotisée.

\section{L'affiche touristique balnéaire : aspects généraux}

L'affiche en tant que support de communication est présente depuis l'Ancien Régime, et ce, sous plusieurs formes : affiches royales, religieuses, privées, commerciales, de spectacle... Cependant, l'affiche touristique apparaît bien au XIX ${ }^{\mathrm{e}}$ siècle et se massifie à partir des années 1880 . Elle bénéficie à ce moment des conjonctures techniques, politiques et artistiques favorables à sa grande diffusion. Elle profite d'abord des progrès techniques et notamment celui concernant le procédé lithographique. Si ce procédé fut découvert vers 1800, son application à l'affiche se développe réellement vers 1830-1840 sous l'impulsion de Jules Chéret (1836-1932). Lithographe de formation, c'est lui qui, le premier, va maîtriser l'affiche en couleurs. Il s'agit d'une technique fondamentale pour la valorisation des stations, puisque désormais les affichistes vont pouvoir jouer sur la coloration de la mer, du ciel, du soleil et des personnages féminins. Les couleurs vives permettent d'attirer l'œil, voire d'éblouir les passants. L'affiche touristique profite ensuite des avancées politiques et de la loi de juillet 1881 sur la liberté d'affichage. Cette loi, voulue par la bourgeoisie industrielle, enlève aux municipalités tous droits de réglementation et proclame la liberté absolue de l'affichage, ce qui a pour conséquence de multiplier le nombre d'affiches. Éditées entre 3000 et 5000 exemplaires, elles sont disposées sur les grands boulevards parisiens, à la gare Saint-Lazare où partent les trains pour la Normandie, dans les gares des stations balnéaires et sur les espaces réservés à l'affichage. Enfin, l'enthousiasme porté par certains artistes reconnus (Pal, Hugo d'Alesi, Sem) pour ce nouveau support artistique favorise l'essor de l'affiche touristique.

Celle-ci provient de différents commanditaires. En premier lieu, nous trouvons les Compagnies des chemins de fer : de l'Est, du Nord, du Midi, de Paris à Orléans, Paris-LyonMarseille et bien sûr de l'Ouest. Cette dernière, créée en 1855 à partir d'une fusion avec de petites compagnies ferroviaires desservant l'Ouest de la France, souhaite promouvoir son réseau ferroviaire et les sites touristiques qu'elle dessert dont les stations balnéaires normandes. C'est pourquoi la mention Chemin de fer de l'Ouest puis de l'État (la Compagnie est rachetée par l'État en 1909 et devient La Compagnie des chemins de fer de l'État puis SNCF en 1938) apparaît sur de nombreuses affiches balnéaires. Il est à noter ici le cas original de Dieppe qui, du fait de sa proximité avec Londres et Paris, devient une escale de la London Brighton \& South Coast Railway qui deviendra la Southern Railway Company en 1923. Cette compagnie qui assure le transport transmanche publie alors des affiches sur Dieppe en anglais et sur le sol anglais. Les syndicats d'initiative sont un autre commanditaire. Si le premier syndicat d'initiative est constitué en France en 1889, il faut attendre les années 1920 pour que ceux du littoral normand se mettent en place. En 1927, on en compte 57 dans toute la Normandie. Sur le littoral nous en trouvons 15 : Blonville-Bénerville, Cabourg, Deauville, Honfleur, Houlgate, Trouville, Villers/mer, Criel-plage, Dieppe, Etretat, Fécamp, Le Havre, Les Petites Dalles, Saint-Valéry-en-Caux, Tréport-Mers. La fonction du syndicat est primordiale puisqu'elle consiste, comme l'écrit en 1927 la revue La Normandie Illustrée, au «développement de la circonscription territoriale ou de la station thermale, climatique ou de tourisme à laquelle [le syndicat] applique son activité en vue d'y améliorer sans cesse les conditions d'accès et de séjour, d'en réaliser le meilleur aménagement, d'y organiser le renseignement sur place et par correspondance et d'en poursuivre la propagande par tous les moyens en son pouvoir». Les syndicats d'initiative éditent, dans cette logique de promotion, de nombreuses affiches et prennent, en ce sens, le relais de la Compagnie de l'État dont les tirages décroissent. Enfin, nous trouvons les affiches émanant des sociétés commerciales comme les casinos ou celles des sociétés sportives comme l'Automobile Club de France. Notons que les événements sportifs, et plus particulièrement les courses automobiles, sont un facteur déterminant dans la renommée et la popularité des stations. Dans cette perspective, Deauville devance toutes les autres en proposant, dès les années 1900, de nombreuses courses automobiles qui attirent une foule considérable. 
L'affiche touristique est un vecteur de communication visant à promouvoir le site balnéaire. Pour ce faire, l'affiche va valoriser la station par la mise en scène de ses atouts touristiques : les paysages (les falaises, la plage, la verdure), les équipements (les bains, les hôtels, les casinos, les sports), l'accessibilité (les horaires de train, les liaisons par bateau puis par avion), le dépaysement (la figure locale, la pêcheuse, le pêcheur, la paysanne), le patrimoine et la femme. Bien évidemment, l'affiche touristique a évolué entre 1880 et 1960 comme la clientèle balnéaire. D'ailleurs, la terminologie utilisée pour nommer les estivants évolue et l'on parlera chronologiquement des baigneurs, des villégiaturistes, des étrangers puis des touristes. Durant cette période, l'activité balnéaire se démocratise et se massifie, bien que certaines stations comme Deauville gardent leur caractère élitiste. Ce qui veut dire que la communication s'est adaptée à ces changements sociaux. On constate d'abord des évolutions graphiques puisque les affiches témoignent des différents mouvements artistiques du moment. Ainsi, l'affiche de la Belle Époque surchargée de textes superlatifs et de vignettes représentant les hôtels, les casinos ou la plage laisse place dans les années 1920 au style Art déco beaucoup plus moderne. L'accent est alors mis sur la géométrie rigoureuse du dessin et sur une pluralité de couleurs. Ce style graphique s'inscrit dans une technique de communication qui se veut plus courte, plus simple, plus percutante. Cassandre, grand affichiste des années 1930, déclarait en 1935 dans ses Notes (Weill, 1995 : 6) : «L'affichiste joue le rôle du télégraphiste : il n'émet pas de message, il les transmet. On ne lui demande pas son avis, on lui demande d'établir une communication claire, puissante, précise...» Cette volonté d'épurer le style et le message s'accélère avec le phénomène de démocratisation de la plage dès la fin des années 1930, époque des premiers congés payés, du camping, du naturisme et du plein air. Ce nouveau public plus populaire, plus local, mais surtout beaucoup plus nombreux incite les stations à s'adapter. Aussi, les affiches valorisent d'avantage la plage, la mer, la baignade et le caractère familial de la station plutôt que le luxe. Exception faite pour Deauville qui gardera ses ambitions élitistes et mondaines.

\section{La mondaine, symbole du tourisme balnéaire}

L'image de la femme est récurrente au sein de notre corpus. Les affiches la montre en baigneuse, en sportive, en mère de famille, dénudée, mais surtout vêtue de longues robes colorées, avec dentelles, chapeaux et ombrelles. Ce sont là les signes caractéristiques de l'aristocratie et de la haute bourgeoisie. En effet, cette femme mise en scène est avant tout une mondaine, c'est-à-dire une femme qui ne travaille pas, une femme qui gouverne son personnel, une femme qui s'adonne aux loisirs de son rang et une femme qui se distingue par ses différentes apparences vestimentaires. Le vêtement porte en ce sens les valeurs et les codes de l'époque. C'est un marqueur social qui témoigne du rang et de la classe sociale. Il s'agit, à travers cette image de la mondaine, d'exprimer l'élégance, la grâce, la beauté et le charme de ce monde balnéaire tout en affichant un monde où le luxe et la richesse s'étalent. Cette représentation affichée de la mondaine suit l'évolution de la mode et des codes sociaux. Aussi, pendant les années folles, apogée du tourisme mondain, la mondaine apparaît sous un nouveau visage : cheveux courts, sans corset, robes courtes, robe-chemise, chapeau cloche... Inspirée par Coco Chanel (elle ouvre une boutique à Deauville en 1913), cette nouvelle mode joue avec les genres. Les femmes arborent polos, cardigans, jodhpurs, costumes et pantalons, autant de signes masculins. Or, cette nouvelle allure cristallise les perceptions contradictoires d'une identité féminine en pleine évolution.

Effectivement, en raccourcissant les jupes, en se coupant les cheveux et en adoptant le costume masculin, la femme devient la garçonne. Pourtant, si cette dernière devient le symbole d'un monde balnéaire en pleine effervescence, il en est autrement dans la réalité, car la garçonne dérange autant qu'elle fascine comme l'explique Christine Bard (1998 : 57) :

Autant qu'une mode qui révolutionne les apparences,

la garçonne représente un nouveau «type social», celui de la femme affranchie. En 1922, Victor Margueritte en donne dans son best-seller La Garçonne un portrait qui provoque l'un des plus grands scandales littéraires du siècle. Non parce qu'il dépeint l'émancipation d'une femme, thème déjà ressassé par la littérature, mais parce qu'il montre une femme de la génération des dancings et du jazz s'adonnant à la drogue et multipliant les expériences sexuelles. La garçonne résume à elle seule la perte des repères dans le monde de l'après[-]guerre et devient la métaphore de la ruine de la civilisation.

Georges Vigarello (2005 : 32-33) conçoit cette métamorphose ainsi :

[Elle] prétend révéler l'autonomie dans les lignes du corps, [...] donne l'indice de transformations qui la dépassent : celles qui révolutionnent la place du féminin dans la société [...] Une femme nouvelle émergerait de ces profils plus actifs. L'illusion d'avoir conquis des droits. Celui au moins de refuser le corset. Celui des grandes enjambées, celui des épaules à l'aise, de la taille qui n'est plus serrée. La mode à la [g]arçonne confirme la mutation.

En effet, pendant la Grande Guerre, les bourgeoises ont acquis une autonomie à laquelle elles ne semblent pas vouloir renoncer. Elles veulent aussi oublier les atrocités de la guerre et, faisant fi des conventions sociales, les bourgeoises sortent, s'amusent, fument.

Toutefois, comme le précise Christine Bard (1998:63), cela cache une réalité sociale complètement différente, car l'idéal de la femme au foyer semble plus que jamais incontesté :

L'apparence, bien sûr, n'est pas seule vérité. Elle a pu donner le change : cacher des «normes traditionnelles demeurées vivaces», de vieux dispositifs de dépendance, un salariat féminin en croissance par exemple, mais rare encore pour les femmes mariées. L'idéal de la femme au foyer semble "plus que jamais incontesté», magnifié par les notables, les moralistes, les médecins.

Aussi cette image de quelques femmes qui transgressent les bonnes mœurs nourrit l'illusion d'une émancipation généralisée pendant ces années folles d'après-guerre. D'ailleurs, ce terme d'années folles, comme l'explique Yvonne Knibiehler (2001 : 26), est à envisager avec circonspection : 
[C]e sont surtout des écrivains et des journalistes d'extrême droite qui ont complaisamment décrit, et pour une part imaginé, une société d'après[-]guerre livrée à la débauche. Dès cette époque, des «observateurs» pour le moins partiaux se lamentent sur l'augmentation du divorce, du célibat volontaire, sur la dissipation sexuelle, le métissage des «races», l'hédonisme qui s'étale dans les dancings ou les fumeries d'opium, le «développement» de l'homosexualité.

En ce sens, l'affiche balnéaire, par la mise en valeur de cette femme mondaine participe à la diffusion d'une image idéalisée de la femme, mais une image quelque peu tronquée puisque cette femme si moderne soit-elle reste marginale.

Les affiches, dans leur souci d'exposer les atouts des stations, promeuvent le casino, et ce, le plus souvent en y associant la femme mondaine. À coté d'une reproduction du casino, souvent en arrière-plan, l'affiche intègre l'image d'une femme dans l'eau ou en dehors. Un autre type d'affiches ne présentent qu'une femme en tenue de bain avec le nom du casino. De fait, la mondaine se reconnaît à ses loisirs, symboles de son rang social. Dans cette perspective, le casino incarne le lieu de loisir privilégié et incontournable, d'autant plus que ces salles de jeux payantes sont une nécessité vitale pour la vie des stations. Le casino devient le cœur de la station, là où il est de bon ton de se montrer. Les gens du monde y viennent lire, jouer (billard, échecs, dames, baccara, petits chevaux et jeux de cartes), danser et écouter des concerts symphoniques ou des spectacles de music-hall. Les promoteurs des casinos, et donc des stations, valorisent par conséquent leur site et leurs infrastructures à travers la femme qui devient une cible «marketing» et par ricochet leurs maris fortunés, ce qui tendrait à confirmer notre hypothèse concernant le rôle central de ces dernières dans le tourisme balnéaire.

Une autre femme est mise en scène dans les affiches, la sportive. Plusieurs affiches montrent une femme jouant au golf, pratiquant l'équitation ou bien en tenue de sportive. C'est le cas d'une affiche concernant la station d'Etretat sur la côte d'Albâtre. On y voit une femme tenant une bicyclette d'une main avec dans l'autre main une raquette de tennis, autre pratique sportive à la mode. Vêtue d'un pantalon et d'un chapeau, elle contemple la mer. Il s'agit bien ici de montrer que la pratique sportive fait partie intégrante de la station et de la vie balnéaire. Il faut dire que le sport joue un rôle fondamental au sein de la sociabilité mondaine balnéaire. À l'aube $\mathrm{du} \mathrm{XX}^{\mathrm{e}}$ siècle, les sports modernes concernent en très grande majorité les aristocrates et les mondaines qui, comme l'indique Catherine Louveau (2006 : 119), «s'adonnent à des loisirs qui sont des passe-temps de la femme «oisive» et des fortunés, pratiques intégratives de classe plus qu'activités sportives à proprement parler». S'il existe derrière cette pratique féminine un discours médical hygiéniste, les bourgeoises sont accusées par les pouvoirs publics et les médecins d'oisiveté et de sédentarité mettant ainsi en danger la régénérescence de la «race». L'élite mondaine prend surtout modèle sur la high life anglaise qui a fait du sport une activité sociale de classe. Cependant, cette mondaine-sportive est vivement critiquée par les partisans d'un sport purement masculin. C'est le cas d'Henri Desgranges qui écrit dans le quotidien sportif L'Auto en juin 1904 :
Il n'est point d'être plus odieux que ce que l'on appelle la femme sportive, celle qui est préoccupée comme nous et presque autant que nous de faire de la marche, du tennis, de l'escrime, du cheval et qui a tant de choses sportives inscrites dans le programme de son existence journalière qu' elle ne trouverait plus le temps de donner à téter à son gosse si elle n'avait point aidé la providence à ne pas lui en donner et qu'elle ne trouve pas davantage celui de songer aux soins de son intérieur et à la décence de sa tenue (p. 1).

Parmi les pratiques sportives stigmatisées, l'usage de la bicyclette rassemble toutes les craintes et les préoccupations non seulement médicales, mais morales de l'époque. À la fin du XIX $^{\mathrm{e}}$ siècle, les bourgeoises adoptent cet exercice perçu comme émancipateur, exercice que la morale tout comme la religion réprouvent. Avec le droit de pédaler, les femmes gagnent le droit à la mobilité, le droit d'aller où elles veulent, quand elles le veulent. Ce droit leur octroie une liberté bien plus tangible, facile et quotidienne (Thompson, 2000). Cette pratique féminine, importée par la clientèle parisienne, se retrouve dans les stations comme l'écrit le journal La Plage Normande en juillet 1896 : «Décidément, ce sport entre de plus en plus dans nos mœurs et toutes les femmes s'y adonnent avec frénésie, délaissant le cheval. De nombreuses cyclowomen sillonnent en tous sens les routes ». Pourtant, ce nouvel acquis pose problème aux médecins, car c'est un engin en pleine diffusion sur lequel de plus en plus de femmes, toutes conditions confondues, montent à califourchon en portant une sorte de pantalon bouffant, jugé plutôt osé. John Carteret, en 1899, dans son ouvrage intitulé La Femme en culotte collecte un grand nombre de caricatures centrées sur la façon dont les femmes qui sortent des "normes vestimentaires» sont représentées comme menaçant, bousculant l'ordre social établi. Ainsi, de nombreux commentateurs de l'époque voient dans la pratique de la bicyclette par les femmes un véritable danger pour l'identité féminine. Le cyclisme pratiqué par ces femmes en culotte est accusé d'abolir les différences de sexe, mais plus encore de créer une génération de femmes viriles et féministes.

Il est certain que le développement de ce sport a fait faire au sexe féminin un pas important dans la voie de son affranchissement, de l'affirmation de sa personnalité. Mais il est certain aussi que le pantalon ou la jupe très courte, assez récemment inaugurés par nos cyclewomen, leur donnent une physionomie jusqu'alors inconnue [...] Cette révolution dans le costume pourrait bien avoir, moralement et sciemment, une origine très grave et beaucoup de personnes de sens estiment que c'est un important épisode de la «revendication féministe». Pour la première fois, sans que la loi puisse garantir à l'homme le monopole, la femme lui dispute l'attribut masculin par excellence : le pantalon (Thompson, $2000: 36$ ).

Malgré ce discours, la femme sportive symbolise une société balnéaire au sein de laquelle les loisirs sportifs occupent une place de plus en plus prépondérante. Toutefois il s'agit de pratiques sportives de classe : tennis, équitation, golf, croquet. 
La communication touristique balnéaire semble donc destinée dès l'origine aux femmes mondaines. Si ce sont bien des hommes d'affaires qui participent à la «balnéarisation» du littoral normand, ce sont surtout leurs femmes qui font vivre ces nouvelles destinations touristiques en y séjournant avec leur personnel près de trois mois. L'essor du réseau ferré entre Paris et la Normandie accélérera ce tourisme de villégiature (Boyer, 2008) et sera à l'origine du développement urbain des stations. Ainsi, chaque saison (de juillet à septembre), les plages normandes voient leur nombre d'habitants se multiplier et se doivent de gérer au mieux ce phénomène touristique. En 1907, 60000 «étrangers» sont ainsi recensés à Trouville, 20000 à Deauville et au Havre, entre 6000 et 8000 à Cabourg, Houlgate et Villers/mer. Vivant dorénavant du pouvoir économique de ces familles aristocratiques et bourgeoises parisiennes, les acteurs économiques locaux cherchent à fidéliser cette clientèle féminine en développant des distractions et des loisirs (sports, promenades, excursions dans les campagnes environnantes, séances de pêche à pied, jeux, casino), si bien que la société balnéaire se manifeste d'une part, à travers ses loisirs de classe et d'autre part, à travers la figure incontournable de la mondaine. C'est pourquoi ces deux éléments fondamentaux de la vie balnéaire se retrouvent exposés sur les affiches.

\section{De la baigneuse à la femme érotisée}

L'affiche n'expose pas seulement une mondaine en tenue de ville, elle nous montre aussi une baigneuse intrépide qui affronte les éléments naturels : la vague, le vent, le froid. La femme est représentée en mouvement, on la voit pratiquer le fameux bain à la lame avec un maître-baigneur ou bien s'exerçant à nager. Sourire aux lèvres, cheveux au vent, décoiffée, loin des mondanités et des préciosités, la baigneuse se présente sans artifices, au naturel, loin des conventions mondaines. Ces affiches, essentiellement celles de la Belle Époque, vantent les vertus thérapeutiques de la mer. Nous sommes là dans un contexte d'inquiétude concernant la baisse de la natalité et la dégénérescence de la «race» d'où cette attention accordée aux agents naturels hygiéniques, tels que l'air et l'eau. Cette nature marine est censée régénérer et oxygéner les corps notamment ceux «fragiles» des femmes. L'établissement de bains, en proposant des bains d'eau chaude et d'eau froide, répond à cette préoccupation médicale. Ceci dit, la mer devient un second espace thérapeutique, c'est le bain à la lame où l'on plonge le baigneur directement dans la vague. La natation devient une activité largement conseillée aux femmes. Le célèbre gynécologue Ernest Monin explique par exemple en 1895 que « [1] a natation [lui] a toujours semblé aussi l'un des plus fidèles agents du développement thoracique régulier : elle surajoute, à des effets musculo-pectoraux utiles, la salutaire influence générale de l'hydrothérapie» (p. 67).

Or, l'image de la baigneuse et de la représentation du corps féminin évolue dans le temps. On passe d'une femme aux formes généreuses, le plus souvent habillée du costume de bain, à un corps libéré des carcans vestimentaires, celle d'un corps plus libre de ses mouvements, celle d'un corps de plus en plus dénudé où les jambes et la poitrine se découvrent progressivement, celle d'un corps qui s'érotise. Cette évolution est liée à la transformation des usages de la mer. Le caractère curatif du bain s'efface progressivement devant le caractère hédoniste et divertissant de la baignade. Ainsi, sous l'influence du mouvement hygiéniste toujours actif, les femmes sont exhortées à la pratique des activités de plein air. La norme est alors au teint hâlé contrairement à la pâleur qui domine les référents esthétiques féminins du siècle précédent. De plus, les bains de mer, la marche, tous ces exercices préconisés par le corps médical d'avant-guerre, imposent eux-aussi une tenue moins contraignante. Libérer le corps devient une nécessité (Vigarello, 2005).

Cette tendance s'accentue à partir des années 1930 jusqu'à l'apparition des pin-up à l'aube des années 1940-1950. On assiste à l'érotisation de la femme et de la baigneuse. Les affiches transposent cette nouvelle représentation du tourisme balnéaire en exhibant de jolies baigneuses aux attitudes suggestives et aguicheuses. Leur corps se dévoile, la baigneuse devient une femme attirante. Les images deviennent plus audacieuses comme en témoigne cette affiche pour Deauville datée de 1956. On y voit au premier plan une femme aux longs cheveux blonds portant un bikini blanc faisant ressortir sa peau hâlée. Étendue langoureusement sous un arbre, elle tient une pomme dans les mains. La référence à l'Ève tentatrice est pour le moins explicite. Deauville serait-elle alors la station de toutes les tentations? Les stations font donc évoluer leur communication en simplifiant leur message et en érotisant la plage. Tout est axé autour de cette femme-érotisée qui s'incarne à travers ses formes parfaites, sa beauté, ses attitudes aguicheuses et ses maillots osés. N'oublions pas que cette représentation féminine est une vision purement masculine puisque tous les affichistes sont des hommes. Nous sommes donc dans une image fantasmée de la femme. Répondant aux critères normatifs de la féminité de l'époque, la femme est représentée avec ses longues jambes, sa fine taille marquée et sa poitrine avantageuse. Comme l'explique très bien Thierry Terret $(2005: 295)$, « [1] a quatrième manière de montrer les sportives passe par une valorisation grossière de leur attractivité. Jambes nues, position ambiguë, insistance lourde sur les anatomies dévoilées signent un parti pris d'érotisation [...] L'érotisation de la femme explique la place étonnamment importante accordée au maillot bain...»

Sa peau devient halée car le bronzage est recommandé. La peau blanchâtre n'est plus de rigueur et apparaît désormais comme un signe maladif lié à l'enfermement. Ce bronzage est d'autant plus facile à dévoiler que les maillots de bain se raccourcissent. Il est intéressant de s'attarder sur cette évolution du maillot de bain comme signe d'évolution des mœurs balnéaires (Saillard, 1998). Au début du tourisme balnéaire, la nudité est interdite. Des codes de plage sont édités afin d'éviter toutes atteintes à la morale. Dans cette logique, les femmes arborent un costume de bain composé de six pièces plus des chaussons. Cette tenue ne laisse rien apparaître du corps. Qui plus est, les hommes et les femmes se baignent séparément. Cette séparation des sexes cesse définitivement à partir des années 1920. En même temps, le maillot se transforme. Les femmes portent un justaucorps similaire à celui des hommes. Ce nouveau maillot, plus simple et plus 
fonctionnel, moule le corps et laisse dévoiler les formes féminines. Désormais les jambes, les bras et la poitrine s'affichent. Par la suite, ce sera l'apparition du bikini.

Cependant, au cours de ces années, l'idéologie dominante cantonne toujours les femmes à leur devoir social et biologique de mère. Pour Françoise Thébaud (1982:151), «[e]xhorter les femmes au devoir de maternité est plus que banal; ligues natalistes, hommes d'Église, médecins, sages-femmes, hommes politiques (jusqu'au centre gauche) s'y emploient à toute occasion; la procréation est en quelque sorte «l'impôt du sang» que le sexe féminin doit à un pays traumatisé par la guerre». La féminité reste associée à la maternité et faire naître demeure une véritable nécessité et une préoccupation majeure durant l'entre-deux-guerre. En définitive, toujours F. Thébaud (1992: 53) le note :

[L]es années d'entre-deux-guerres apparaissent [...], dans l'histoire des femmes, comme une époque de transition entre modernité esquissée et traditions réaffirmées. L'image valorisée est celle de la femme, naturellement fragile, assurant la fonction de mère de famille, d'épouse et de gardienne de foyer. Les mentalités n'ont pas été profondément modifiées. La guerre a plutôt tendance à fortifier la pensée sociale avant 1914, qui de façon quasi consensuelle, faisait de la travailleuse un être intrinsèquement faible et de la femme une «mère de la race» avant tout.

Ainsi, malgré cette vision, la baigneuse ou la mondaine demeurent avant tout des femmes, garante de l'avenir de la «race». Les discours savants de l'époque se structurent sur l'idée forte que la fonction sociale et biologique des femmes est avant tout l'enfantement, sa «nature» autant que son devoir, ce rôle étant plus ou moins exclusif de tout autre. C'est la «nature» qui établit les règles sociales (Guillaumin, 1992) et les rôles attribués socialement aux femmes deviennent des faits «naturels». Le processus de naturalisation de la femme se base sur des fondements scientifiques forts et prend appui sur une association progressive faite entre femmes et reproduction. Yannick Ripa (2002 : 17-18) explique :

Les connaissances sur la génération, pourtant balbutiantes, concluent que toute l'économie féminine a pour unique fonction la maternité : la matrice résume la femme. Ainsi glisse-t-on du destin à la destinée. Qu'elle soit œuvre de Dieu ou de la [n] ature, la femme se confond avec la mère et n'a d'autre raison d'être. L'idée reçue en fait un réceptacle de la semence masculine, un ventre qui doit porter l'enfant, définition peu propice à l'exaltation de la maternité.

Les représentations sociales rattachent la femme à son rôle de mère reproductrice et nourricière. Enfanter est donc sa fonction sociale, son destin individuel. Comme le rappelle Geneviève Fraisse (1998 : 56), «[e]ngendrer est sa «destination naturelle», souligne Virey, et l'étymologie, la sienne, sert de preuve aussi abruptement que l'argument autoritaire de la nature : femme, foemina, foetare, fotus, ont une même racine linguistique». Il n'est donc pas étonnant que la femme-mère soit représentée sur certaines affiches touristiques.

De fait, l'affiche balnéaire qui, comme nous l'avons évoqué, s'adresse directement aux femmes met en scène une mère avec ses enfants, le plus souvent un garçon et une fille. Dès les années 1880 , la mère de famille est présente sur les affiches. Le plus souvent en tenue mondaine, elle veille d'un regard bienveillant sur ses enfants jouant sur le sable avec pelle et seau, premiers jouets balnéaires. Dans les années 1950, nous retrouvons cette même femme-mère avec ses enfants. Ce qui veut dire que cette représentation, au-delà du discours social dominant, sert aussi d'argument pour les stations. La plage devient au fil des années un lieu de loisirs destinés aux femmes et surtout à leurs enfants, c'est la création des clubs de plages. D'ailleurs, par la suite certaines stations comme Trouville axeront leur communication exclusivement sur ce thème de plage familiale, royaume des enfants, bien loin des fastes de sa voisine Deauville.

\section{Conclusion}

D’après notre analyse, la présence féminine sur les affiches répond clairement à une intention publicitaire et promotionnelle. Toutefois, au sein de cette stratégie générale, il est nécessaire de distinguer deux niveaux d'analyse. L'image féminine doit d'abord charmer le touriste homme, celui qui possède l'argent, en lui donnant l'envie de voyager et de venir dans une station. Qui mieux alors qu'une jolie élégante dévêtue se baignant pour exhorter cette envie de faire partie de l'aristocratie balnéaire? En ce sens, les femmes incarnent un atout de promotion et un argument marketing fondamental pour les stations. Cependant, l'affiche s'adresse également aux femmes elles-mêmes, car ces femmes mondaines incarnent ce tourisme balnéaire sédentaire. Ce sont elles qui sont présentes, avec les enfants et leurs domestiques, pendant la période estivale alors que les maris qui gèrent leurs affaires ne restent pas. Ce qui signifie que les femmes sont les principales actrices de ce tourisme et de ce monde balnéaire. Apposer une femme sur les affiches semble donc naturel et logique tant les femmes symbolisent cette réalité touristique.

Or, comme nous l'avons vu, la représentation de ces femmes s'avère être surtout une vision masculine fantasmée, éloignée des réalités sociales de l'époque. Si de par sa nudité, ses poses suggestives, l'image incarne une femme libérée, émancipée, il en est autrement dans les faits puisque la femme demeure soumise aux codes moraux, religieux et sociaux. L'affiche touristique apparait donc comme un outil de promotion du site balnéaire, mais en l'idéalisant et en le sublimant. De fait, cette stratégie se retrouve sur les affiches balnéaires des autres littoraux notamment pour la côte d'Azur. La femme incarne donc véritablement le tourisme balnéaire et en devient le symbole.

\section{Références}

ARNAUD, Pierre et Thierry TERRET (2007) Histoire du sport féminin (t. 2), Paris : L'Harmattan. $270 \mathrm{p}$.

BARD, Christine (1998) Les garçonnes, modes et fantasmes des Années folles, Paris : Flammarion. 159 p.

BLANQUET, Albert (1859) Les bains de mer des côtes normandes, Paris : Hachette. 125 p.

BOYER, Marc (2003) Le tourisme en France, Paris : Éditions EMS. 291 p.

BOYER, Marc (2008) Les villégiatures du XVI a a XXIe siècle. Panorama du tourisme sédentaire, Paris : Éditions EMS. 238 p. 
BUSSI, Michel (2007) «La côte d'Albâtre: heurs et malheurs touristiques depuis deux siècles", Les Études Normandes, no 3, p. 37-50.

CARTERET, John (1899) La femme en culotte (réédition en 1993), Paris : Éditions Côté-Femmes. 167 p.

CASSANDRE (1935) Notes, cité dans WEILL, Alain (1995) Les maîtres de l'affiche-Cassandre, Paris : Éditions Bibliothèque de l'Image. 64 p.

CORBIN, Alain (1990) Le Territoire du vide. L'Occident et le désir du rivage, 1750-1840, Paris : Flammarion. 407 p.

CORBIN, Alain (1995) L'avènement des loisirs (1850-1960), Paris : Flammarion. 466 p.

DESERT, Gabriel (1983) La vie quotidienne sur les plages normandes du Second Empire aux années folles, Paris : Hachette. 356 p.

DESGRANGES (12 juin 1904) L'Auto, éditorial, p. 1.

FRAISSE, Geneviève (1998) Les femmes et leur histoire, Paris : Gallimard. 614 p.

GUILLAIN, Jean-Yves (2007) «Villégiature et nouveaux espaces sportifs : images de la sportive dans les affiches touristiques de l'entre-deux-guerres », chapitre 2 DANS Sport et Genre, sous la direction de Anne ROGER et Thierry TERRET, p. 121-135. Paris : L'Harmattan.

GUILLAUMIN, Colette (1992) Sexe, race et pratique du pouvoir. L'idée de Nature, Paris : Éditions Côté-Femmes. 239 p.

GUILLET, François (2000) «Les guides consacrés à la Normandie pendant la première moitié du XIXe siècle (1815-1850)», chapitre 36 DANS Les guides imprimés du XVI au XXe siècle, villes, paysages, voyages, sous la direction de Gilles CHABAUD, Evelyne COHEN, Natacha COQUERY et Jérôme PENEZ, p. 501-511. Belin : Paris.

HERVIEU, Jean-Paul; Gilles DESIRE DIT GOSSET et Eric BARRE (2002)

Bains de mer et thermalisme en Normandie, Condé/Noireau : Éditions Charles Corlet. 245 p.

KNIBIEHLER, Yvonne (2001) «Politique et maternité : sous Vichy, la procréation est un enjeu national, patriotique donc politique», DANS Combats de femme, 1939-1945. Françaises et Allemandes, les oubliées de l'histoire, sous la direction d'Evelyne MORIN-ROTUREAU, p. 49-63. Paris : Autrement.

LANQUAR, Robert et Robert HOLLIER (1996) Le marketing touristique, Paris : PUF. 127 p.

LA PLAGE NORMANDE (1896) Organe des stations balnéaires, Caen : Rédaction et administration.

L’ÉCHO DES PLAGES (1892-1914) Journal balnéaire, Caen : Rédaction et administration.

LORIS (de), C. (1896) La femme à bicyclette. Ce qu'elles en pensent, Paris : May et Motteroz. 37 p.

LOUVEAU, Catherine (2006) «Inégalité sur la ligne de départ : femmes, origines sociales, conquête du sport ", Clio, no 24, p. 119-143.

MONIN, Ernest (1895) La santé de la Femme (entretiens sur l'hygiène et la médecine féminines), Paris : Octave Doin. 300 p.
MORIÈRE, Emile (dir.) (1910-1914) La Revue illustrée du Calvados : publication mensuelle des faits et événements de tout le département, Lisieux : Imprimerie E. Morière.

PALMAERT (de), Albéric (2005) Un siècle de bords de mer (1850-1950), Rennes : Éditions Ouest-France. 122 p.

PECOUT, Christophe et Ludovic BIROT (2008) «La culture sportive à la Belle-Époque : facteur du développement des stations balnéaires du Calvados ", Les Annales de Normandie, n 1 et 2, p. 135-147.

PIC, Rafael (2009) L'Europe des bains de mer, Paris : Éditions Nicolas Chaudin. 253 p.

POULLET, Ginette (2006) Au vrai chic balnéaire. Petits échos des plages normandes de 1806 à 1929, Condé-sur-Noireau : Éditions Charles Corlet. $209 \mathrm{p}$.

RAUCH, André (1993) Les Vacances, Paris : PUF. 125 p.

RIPA, Yannick (2002) Les femmes, actrices de l'Histoire. France, 1789-1945, Paris : Sedes. 192 p.

SAILLARD, Olivier (1998) Les Maillots de bain, Paris : Éditions du Chêne. $128 \mathrm{p}$.

SOHN, Anne-Marie (2002) «Entre-deux-guerres, les rôles féminins en France et en Angleterre», Chapitre 3 DANS Histoire des femmes en occident : Le $20^{e}$ siècle, sous la direction de Georges DUBY, Michelle PERROT et Françoise THÉBAUD, p. 105-132. Paris : Perrin.

TERRET, Thierry (2005) «Sport et genre dans la presse sportive des années cinquante. L'exemple de Sport \& Vie», Chapitre 12 DANS Sport et genre, vol. 2, sous la direction de Philippe LIOTARD et Thierry TERRET, p. 263-297. Paris : L’Harmattan.

THÉBAUD, Françoise (1982) «Donner la vie : histoire de la maternité en France entre les deux guerres». Thèse de doctorat en histoire sous la direction de Michèle PERROT, Paris : université de Paris 7.

THÉBAUD, Françoise (1992) «La grande guerre. Le triomphe de la division sexuelle», Chapitre 1 DANS Histoire des femmes en Occident. Le $20^{\text {ème }}$ siècle, sous la direction de Georges DUBY, Michèle PERROT et Françoise THÉBAUD, p. 31-74. Paris : Plon.

THOMPSON, Christopher (2000) «Un troisième siècle? Les bourgeoises et la bicyclette dans la France fin de siècle», Le mouvement social, n 192, p. 9-39.

VATTIER D'AMBROYSE, Valentine (1891) Le littoral de la France. Côtes normandes, Paris : Champion-Slatkine. $182 \mathrm{p}$.

VIGARELLO, Georges (2005) «Années folles : le corps métamorphosé», Sciences humaines, $\mathrm{n}^{\circ} 162$, p. 32-35.

WEILL, Alain (1995) Les maîtres de l'affiche-Cassandre, Paris : Éditions Bibliothèque de l'Image. 64 p.

WOLF, Pierre-André (dir.) (1926-1928) La Normandie illustrée. Revue de tourisme et d'art : Rouen. 